TRANSACTIONS OF THE

AMERICAN MATHEMATICAL SOCIETY

Volume 193, 1974

\title{
ON TOTALLY REAL SUBMANIFOLDS
}

BY

\section{BANG-YEN CHEN( ${ }^{1}$ ) AND KOICHI OGIUE(2)}

ABSTRACT. Complex analytic submanifolds and totally real submanifolds are two typical classes among all submanifolds of an almost Hermitian manifold. In this paper, some characterizations of totally real submanifolds are given. Moreover some classifications of totally real submanifolds in complex space forms are obtained.

1. Introduction. Among all submanifolds of an almost Hermitian manifold, there are two typical classes: one is the class of bolomorphic submanifolds and the other is the class of totally real submanifolds. A submanifold $M$ of an almost Hermitian manifold $\widetilde{M}$ is called bolomorphic (resp. totally real) if each tangent space of $M$ is mapped into itself (resp. the normal space) by the almost complex structure of $\tilde{M}$. There have been many results in the theory of holomorphic submanifolds; on the other hand, there have been only a few results in the theory of totally real submanifolds.

The purpose of this paper is to study some fundamental properties of totally real submanifolds.

In $\$ 2$, we investigate the general properties of totally real submanifolds.

In $\$ \$ 3$ and 4 we consider totally real submanifolds of a complex space form.

In the last section, we first give a characterization for totally real surfaces in a complex space form, later we prove some results for totally real or holomorphic surfaces by using analytic function theory.

2. Totally real submanifolds. Let $M$ be an $n$-dimensional Riemannian manifold and $\tilde{M}$ be a Kaehler manifold of dimension $2(n+p), p \geq 0$. Let $\tilde{J}$ be the almost complex structure of $\widetilde{M}$ and let $g$ (resp. $\tilde{g}$ ) be the Riemannian metric of $M$

Received by the editors April 6, 1973.

AMS (MOS) subject classifications (1970). Primary 53B25, 53C40; Secondary 53A10, 53B35.

Key words and phrases. Totally real submanifolds, minimal submanifolds, complex space forms, sectional curvature, scalar curvature.

(1) Work done under partial support by NSF Grant No. 36684.

(2) Work done under partial support by the Matsunaga Science Foundation. 
(resp. $\tilde{M}$ ). We call $M$ an totally real submanifold of $\tilde{M}$ if $M$ admits an isometric immersion into $\tilde{M}$ such that for all $x, \tilde{J}\left(T_{x}(M)\right) \subset \nu_{\boldsymbol{x}}$, where $T_{\boldsymbol{x}}(M)$ denotes the tangent space of $M$ at $x$ and $\nu_{x}$ the normal space at $x$.

By a plane section we mean a 2-dimensional linear subspace of a tangent space. A plane section $\tau$ is called antibolomorpbic if $\tilde{J} \tau$ is perpendicular to $r$.

Proposition 2.1. Let $M$ be a submanifold immersed in an almost Hermitian manifold $\tilde{M}$. Then $M$ is a totally real submanifold of $\tilde{M}$ if and only if every plane section of $M$ is antibolomorpbic.

Proof. Let $X$ be an arbitrary vector in $T_{x}(M)$, and let $e_{1}=X, e_{2}, \ldots, e_{n}$ be a basis of $T_{\boldsymbol{x}}(M)$. We denote by $\boldsymbol{r}_{i j}$ the plane section spanned by $\boldsymbol{e}_{i}$ and $\boldsymbol{e}_{j} \cdot$

Assume that every plane section is antiholomorphic. Then $\tilde{J} r_{1 j}$ are perpendicular to $r_{1 j}$ for $j=2, \ldots, n$. Therefore $\tilde{J} X$ is perpendicular to $e_{1}, e_{2}, \ldots, e_{n}$ so that $\tilde{J} X \in \nu_{x}$. This implies that $M$ is a totally real submanifold of $\widetilde{M}$. The converse is clear. Q.E.D.

Assume that $M$ is a totally real submanifold immersed in $\tilde{M}$. We denote by $\nabla$ (resp. $\widetilde{\nabla}$ ) the covariant differentiation with respect to $g$ (resp. $\tilde{g}$ ). Then the second fundamental form $\sigma$ of the immersion is given by $\sigma(X, Y)=\widetilde{\nabla}_{X} Y-\nabla_{X} Y$. For a normal vector field $\xi$ on $M$, we write $\widetilde{\nabla}_{X} \xi=-A_{\xi} X+D_{X} \xi$, where $-A_{\xi} X$ (resp. $D_{X} \xi$ ) denotes the tangential (resp. normal) component of $\widetilde{\nabla}_{X} \xi$. Then we have

$$
\tilde{g}(\sigma(X, Y), \xi)=g\left(A_{\xi} X, Y\right)
$$

A normal vector field $\xi$ on $M$ is said to be parallel if $D_{X} \xi=0$ for any tangent vector $X$. The mean curvature vector $H$ is defined by $H=(1 / n)$ trace $\sigma$. A submanifold $M$ is said to be minimal if $H=0$ identically. For the second fundamental form $\sigma$, we define the covariant differentiation $\nabla^{\prime}$ with respect to the connection in (tangent bundle) $\oplus$ (normal bundle) by

$$
\left(\nabla_{X}^{\prime}\right)(Y, Z)=D_{X}(\sigma(Y, Z))-\sigma\left(\nabla_{X} Y, Z\right)-\sigma\left(Y, \nabla_{X} Z\right)
$$

for all tangent vector fields $X, Y$, and $Z$ on $M$.

Let $\widetilde{R}$ denote the curvature tensor field of $\tilde{M}$. Then the equation of Codazzi is given by

$$
(\tilde{R}(X, Y) Z)^{\perp}=\left(\nabla_{X}^{\prime} \sigma\right)(Y, Z)+\left(\nabla_{Y}^{\prime} \sigma\right)(X, Z),
$$

where $(\tilde{R}(X, Y) Z)^{\perp}$ denotes the normal component of $\tilde{R}(X, Y) Z$.

We choose a local field of orthonormal frames

$$
\begin{aligned}
e_{1}, \ldots, e_{n^{\prime}} e_{n+1}, \ldots, e_{n+p}, e_{1^{*}} & =\tilde{J} e_{1}, \ldots, e_{n^{*}}=\tilde{J} e_{n^{\prime}} e_{(n+1)^{*}} \\
& =\tilde{J} e_{n+1}, \ldots, e_{(n+p)^{*}}=\tilde{J} e_{n+p}
\end{aligned}
$$


in $\tilde{M}$ in such a way that, restricted to $M, e_{1}, \ldots, e_{n}$ are tangent to $M$. With respect to this frame field, $\tilde{J}$ has the component( 3 )

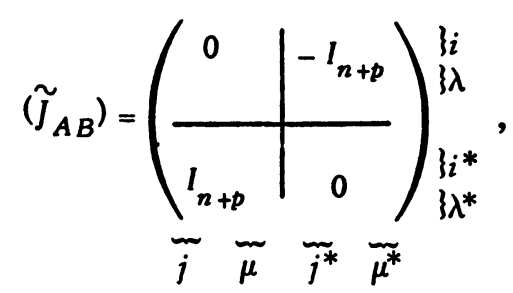

where $I_{n+p}$ denotes the identity matrix of degree $n+p$. If we set $A_{\alpha}=A_{e_{\alpha}}$ i.e., $\sigma(X, Y)=\Sigma g_{\alpha}\left(A_{\alpha}, Y\right) e_{\alpha}$, then the $A_{\alpha}$ 's are local fields of symmetric linear transformations.

With respect to the frame field of $\tilde{M}$ chosen above, let

$$
\omega^{1}, \cdots, \omega^{n}, \omega^{n+1}, \cdots, \omega^{n+p}, \omega^{1 *}, \cdots, \omega^{n *}, \omega^{(n+1) *}, \cdots, \omega^{(n+p) *}
$$

be the field of dual frames. Then the structure equations of $\tilde{M}$ are given by

$$
\begin{gathered}
d \omega^{A}=-\sum \omega_{B}^{A} \wedge \omega^{B}, \\
\omega_{B}^{A}+\omega_{A}^{B}=0 ; \\
\omega_{j}^{i}+\omega_{i}^{j}=0, \quad \omega_{j}^{i}=\omega_{j^{*}}^{i^{*}}, \quad \omega_{j}^{i^{*}}=\omega_{i}^{j^{*}}, \\
\omega_{\mu}^{\lambda}+\omega_{\lambda}^{\mu}=0, \quad \omega_{\mu}^{\lambda}=\omega_{\mu^{*}}^{\lambda^{*}}, \quad \omega_{\mu}^{\lambda^{*}}=\omega_{\lambda}^{\mu^{*}}, \\
\omega_{\lambda}^{i}+\omega_{i}^{\lambda}=0, \quad \omega_{\mu}^{i}=\omega_{\mu^{*}}^{i^{*}}, \quad \omega_{\lambda}^{i^{*}}=\omega_{i}^{\lambda^{*}} ; \\
d \omega_{B}^{A}=-\sum \omega_{C}^{A} \wedge \omega_{B}^{C}+\tilde{\Omega}_{B}^{A}, \quad \tilde{\Omega}_{B}^{A}=1 / 2 \sum \tilde{R}_{B C D^{A}}{ }^{C} \wedge \omega^{D} .
\end{gathered}
$$

Restricting these forms to $M$, we have the structure equations of the immersion:

$$
\begin{gathered}
\omega^{a}=0, \\
d \omega^{i}=-\sum \omega_{k}^{i} \wedge \omega^{k}, \\
d \omega_{j}^{i}=-\sum \omega_{k}^{i} \wedge \omega_{j}^{k}+\Omega_{j}^{i}, \quad \Omega_{j}^{i}=1 / 2 \sum R_{j k l}^{i} \omega^{k} \wedge \omega^{l}, \\
\Omega_{j}^{i}=\tilde{\Omega}_{j}^{i}-\sum \omega_{a}^{i} \wedge \omega_{j}^{a} \text { (the equation of Gauss). }
\end{gathered}
$$

(3) We use the following convention on the range of indices unless otherwise stated: $A, B, C, D=1, \ldots, n+p, 1^{*}, \ldots,(n+p)^{*} ; h, i, j, k, l, m=1, \ldots, n ; a, \beta=n+1, \ldots$, $n+p, 1^{*}, \ldots,(n+p)^{*} ; \lambda, \mu=n+1, \cdots, n+p$. 
From (2.2) and (2.5) we have $\Sigma \omega_{i}^{a} \wedge \omega^{i}=0$. By Cartan's lemma we may write

$$
\omega_{i}^{a}=\sum h_{i j}^{a} \omega^{\prime}, \quad b_{i j}^{a}=b_{j i}^{a} .
$$

We can easily see

$$
b_{i j}^{a}=g\left(A_{a} e_{i}, e_{j}\right)
$$

The equation of Gauss is written as

$$
R_{j k l}^{i}=\tilde{R}_{j k l}^{i}+\sum\left(b_{i k}^{a} b_{j l}^{a}-b_{i l}^{a} b_{j k}^{a}\right) .
$$

3. Totally real submanifolds immersed in a complex space form. A Kaehler manifold of constant holomorphic sectional curvature is called a complex space form. Let $\tilde{M}_{n+p}(\tilde{c})$ be an $(n+p)$-dimensional complex space form of constant holomorphic sectional curvature $\tilde{c}$. Then we have

$$
\tilde{R}_{B C D}^{A}=1 / 4 \tilde{c}\left(\delta_{A C} \delta_{B D}-\delta_{A D} \delta_{B C}+\tilde{J}_{A C} \tilde{J}_{B D}-\tilde{J}_{A D} \tilde{J}_{B C}+2 \tilde{J}_{A B} \tilde{J}_{C D}\right) \text {. }
$$

Let $\tilde{R}$ be the curvature tensor field of $\tilde{M}_{n+p}(\tilde{c})$ so that $\tilde{R}_{B C D}^{A}=\tilde{g}\left(R\left(e_{C}, e_{D}\right) e_{B}, e_{A}\right)$. Then (3.1) is equivalent to

$$
\begin{aligned}
\tilde{R}(\tilde{X}, \tilde{Y}) \tilde{Z}=1 / 4 \tilde{c}\{\tilde{g}(\tilde{Y}, \tilde{Z}) \tilde{X}-\tilde{g}(\tilde{X}, \tilde{Z}) \tilde{Y}+\tilde{g}(\tilde{J} \tilde{Y}, \tilde{Z}) \tilde{J} \tilde{X} \\
-\tilde{g}(\tilde{J} \tilde{X}, \tilde{Z}) \tilde{J} \tilde{Y}+2 \tilde{g}(\tilde{X}, \tilde{J} \tilde{Y}) \tilde{J} \tilde{Z}\},
\end{aligned}
$$

where $\tilde{X}, \tilde{Y}$ and $\tilde{Z}$ are vector fields on $\tilde{M}_{n+p}(\tilde{c})$.

A submanifold $M$ of $\tilde{M}_{n+p}(\tilde{c})$ is called an invariant submanifold if each tangent space is invariant under the curvature transformation: $(\tilde{R}(X, Y)) T_{x}(M) \subset$ $T_{x}(M)$ for all vector fields $X$ and $Y$ on $M$ (cf. [4]).

Proposition 3.1. Let $M$ be an n-dimensional submanifold immersed in $\tilde{M}_{n+p}(\tilde{c})$ with $\tilde{c} \neq 0$. Then $M$ is a bolomorpbic or a totally real submanifold of $\tilde{M}_{n+p}(\tilde{c})$ if and only if $M$ is an invariant submanifold.

Proof. Let $X$ and $Y$ be two vector fields on $M$ and $Z \in T_{x}(M)$. From (3.1)' we have

$$
\begin{aligned}
\tilde{R}(X, Y) Z=1 / 4 \tilde{c}\{g(Y, Z) X-g(X, Z) Y+\tilde{g}(\tilde{J} Y, Z) \tilde{J} X-\tilde{g}(\tilde{J} X, Z) \tilde{J} Y \\
+2 \tilde{g}(X, \tilde{J} Y) \tilde{J} Z\} .
\end{aligned}
$$

If $M$ is a holomorphic or a totally real submanifold, then $\tilde{R}(X, Y) Z \in T_{\boldsymbol{x}}(M)$ is clear.

Conversely, assume that $\tilde{R}(X, Y) Z \in T_{\boldsymbol{x}}(M)$ for arbitrary $X, Y$ and $Z$. Putting $Z=X$, we have 


$$
\tilde{R}(X, Y) X=1 / 4 \tilde{c}\{g(Y, X) X-g(X, X) Y+3 \tilde{g}(X, \tilde{J} Y) \tilde{j} X\} .
$$

Since $\tilde{R}(\underset{\sim}{X}, Y) X \in T_{x}(M)$, we have $\tilde{g}(X, \tilde{J} Y) \tilde{J} X \in T_{x}(M)$ so that either $\tilde{J} X \in T_{x}(M)$ or $\tilde{g}(X, \tilde{J} Y)=0$. This implies that either $\tilde{J}\left(T_{x}(M)\right) \subset T_{x}(M)$ or $\tilde{J}\left(T_{x}(M)\right) \subset \nu_{x}$, i.e., either $M$ is holomorphic or totally real. Q.E.D.

Let $M$ be an $n$-dimensional totally real submanifold immersed in $\tilde{M}_{n+p}(\tilde{c})$. From (2.1), (2.8)' and (3.1) we have

$$
R_{j k l}^{i}=1 / 4 \tilde{c}\left(\delta_{i k} \delta_{j l}-\delta_{i l} \delta_{j k}\right)+\sum\left(b_{i k}^{a} b_{j l}^{a}-b_{i l}^{a} b_{j k}^{a}\right) .
$$

Let $R$ be the curvature tensor field of $M$ so that $R_{j k l}^{i}=g\left(R\left(e_{k}, e_{l}\right) e_{j}, e_{i}\right)$. Then we have

$$
\begin{aligned}
g(R(X, Y) Z, W)= & 1 / 4 \tilde{c}\{g(X, W) g(Y, Z)-g(X, Z) g(Y, W)\} \\
& +\tilde{g}(\sigma(X, W), \sigma(Y, Z))-\tilde{g}(\sigma(X, Z), \sigma(Y, W)) \\
= & 1 / 4 \tilde{c}\{g(X, W) g(Y, Z)-g(X, Z) g(Y, W)\} \\
& +\sum\left\{g\left(A_{a} X, W\right) g\left(A_{a} Y, Z\right)-g\left(A_{a} X, Z\right) g\left(A_{a} Y, W\right)\right\} .
\end{aligned}
$$

This implies that the sectional curvature $K$ of $M$ determined by orthonormal vectors $X$ and $Y$ is given by

$$
\begin{aligned}
K(X, Y) & =1 / 4 \tilde{c}+\tilde{g}(\sigma(X, X), \sigma(Y, Y))-\|\sigma(X, Y)\|^{2} \\
& =1 / 4 \tilde{c}+\sum\left\{g\left(A_{a} X, X\right) g\left(A_{a} Y, Y\right)-g\left(A_{a} X, Y\right)^{2}\right\} .
\end{aligned}
$$

Therefore we have

Proposition 3.2. Let $M$ be an n-dimensional totally real submanifold immersed in $\tilde{M}_{n+p}(\tilde{c})$. If $M$ is totally geodesic in $\tilde{M}_{n+p}(\tilde{c})$, then $M$ is a space of constant curvature $(K=1 / \tilde{c} \tilde{c})$.

Let $S$ be the Ricci tensor of $M$. Then we have

(3.4) $S(X, Y)=1 / 4(n-1) \tilde{c} g(X, Y)+\sum\left(\operatorname{tr} A_{a}\right) g\left(A_{a} X, Y\right)-\sum g\left(A_{a} X, A_{a} Y\right)$.

Let $\rho$ be the scalar curvature of $M$. Then we have

$$
\rho=1 / 4 n(n-1) \tilde{c}+\sum\left(\operatorname{tr} A_{a}\right)^{2}-\|\sigma\|^{2},
$$

where $\|\sigma\|$ is the length of the second fundamental form $\sigma$ of the immersion so that

$$
\|\sigma\|^{2}=\sum \operatorname{tr} A_{a}^{2}=\sum b_{i j}^{a} b_{i j}^{a} \text {. }
$$


If $M$ is a minimal submanifold so that $\operatorname{tr} A_{\alpha}=0$ for all $\alpha$, then we have

$$
\begin{gathered}
S(X, Y)=1 / 4(n-1) \tilde{c} g(X, Y)-\sum g\left(A_{a} X, A_{a} Y\right), \\
\rho=1 / 4 n(n-1) \tilde{c}-\|\sigma\|^{2} .
\end{gathered}
$$

As an immediate consequence of $(3.4)_{m}$ and $(3.5)_{m}$ we have

Proposition 3.3. Let $M$ be an n-dimensional totally real minimal submanifold immersed in $\tilde{M}_{n+p}(\tilde{c})$. Then

(a) $S-1 / 4(n-1) \tilde{c} g$ is negative semidefinite,

(b) $\rho \leq 1 / 4 n(n-1) \tilde{c}$.

The following is also clear.

Proposition 3.4。 Let $M$ be an n-dimensional totally real minimal submanifold immersed in $\tilde{M}_{n+p}(\tilde{c})$. Then $M$ is totally geodesic if and only if $M$ satisfies one of the following conditions:

(a) $K=1 / 4 \tilde{c}$,

(b) $S=1 / 4(n-1) \tilde{c} g$,

(c) $\rho=1 / 4 n(n-1) \tilde{c}$.

The second fundamental form $\sigma$ of the immersion satisfies a differential equation:

Proposition 3.5. Let $M$ be an n-dimensional totally real minimal submanifold immersed in $\tilde{M}_{n+p}(\tilde{c})$. Then

$$
\begin{aligned}
1 / 2 \Delta\|\sigma\|^{2}= & \left\|\nabla^{\prime} \sigma\right\|^{2}+\sum \operatorname{tr}\left(A_{a} A_{\beta}-A_{\beta} A_{a}\right)^{2}-\sum\left(\operatorname{tr} A_{a} A_{\beta}\right)^{2}+1 / 4 \tilde{n c}\|\sigma\|^{2}+1 / 4 \tilde{c} \sum \operatorname{tr} A_{i^{*}}^{2} \\
= & \left\|\nabla^{\prime} \sigma\right\|^{2}+2 \sum \operatorname{tr}\left(A_{a} A_{\beta}\right)^{2}-2 \operatorname{tr}\left(\sum A_{a}^{2}\right)^{2} \\
& -\sum\left(\operatorname{tr} A_{a} A_{\beta}\right)^{2}+1 / 4 n \tilde{c}\|\sigma\|^{2}+1 / 4 \tilde{c} \sum \operatorname{tr} A_{i^{*}}^{2} .
\end{aligned}
$$

Proof. Since $M$ is a minimal submanifold of $\tilde{M}_{n+p}(\tilde{c})$, the following holds [1]:

$$
\begin{aligned}
1 / 2 \Delta\|\sigma\|^{2}= & \left\|\nabla^{\prime} \sigma\right\|^{2}+\sum \operatorname{tr}\left(A_{a} A_{\beta}-A_{\beta} A_{a}\right)^{2}-\sum\left(\operatorname{tr} A_{a} A_{\beta}\right)^{2} \\
& +\sum\left(4 \tilde{R}_{\beta i j}^{a} b_{j k}^{a} b_{i k}^{\beta}-\tilde{R}_{k \beta k}^{a} b_{i j}^{a} b_{i j}^{\beta}+2 \tilde{R}_{j k j}^{i} b_{i l}^{a} b_{k l}^{a}+2 \tilde{R}_{j k l}^{i} b_{i l}^{a} b_{j k}^{a}\right) .
\end{aligned}
$$

Since $\tilde{M}_{n+p}(\tilde{c})$ is a complex space form of constant holomorphic sectional curvature $\tilde{c}$ so that $\widetilde{R}_{B C D}^{A}$ is given by (3.1), the last term of the right-hand side of the above equation is equal to $1 / 4 n \tilde{c}\|\sigma\|^{2}+1 / 4 \tilde{c} \Sigma$ tr $A_{i}^{2}$. Q. E. D. 
Corollary 3.6. Let $M$ be an n-dimensional totally real minimal submanifold immersed in $\tilde{M}_{n}(\tilde{c})$. 'Then

$$
\begin{aligned}
1 / 2 \Delta\|\sigma\|^{2} & =\left\|\nabla^{\prime} \sigma\right\|^{2}+\sum \operatorname{tr}\left(A_{i^{*}} A_{j^{*}}-A_{j^{*}} A_{i^{*}}\right)^{2}-\sum\left(\operatorname{tr} A_{i^{*}} A_{j^{*}}\right)^{2}+1 / 4(n+1) \tilde{c}\|\sigma\|^{2} \\
& =\left\|\nabla^{\prime} \sigma\right\|^{2}+2 \sum \operatorname{tr}\left(A_{i^{*}} A_{j^{*}}\right)^{2}-3 \operatorname{tr}\left(\sum A_{i^{*}}^{2}\right)^{2}+1 / 4(n+1) \tilde{c}\|\sigma\|^{2} \\
& =\left\|\nabla^{\prime} \sigma\right\|^{2}+2 \sum \operatorname{tr}\left(A_{i^{*}} A_{j^{*}}\right)^{2}-3 \sum\left(\operatorname{tr} A_{i^{*}} A_{j^{*}}\right)^{2}+1 / 4(n+1) \tilde{c}\|\sigma\|^{2} .
\end{aligned}
$$

Proof. The first equality is an immediate consequence of Proposition 3.5. The second and the third equalities follow from Proposition 3.5 and Lemma 3.7 (1). Q.E.D.

Lemma 3.7. Let $M$ be an n-dimensional totally real minimal submanifold immersed in $\tilde{M}_{n}(\tilde{c})$. Then

(1) $\operatorname{tr}\left(\Sigma A_{i}^{2}\right)^{2}=\Sigma\left(\operatorname{tr} A_{i} * A_{j}\right)^{2}$.

(2) $\operatorname{tr} A_{i}{ }^{*} A_{j}^{i}=\|\sigma\|^{2} \delta_{i j} / n$ provided that $M$ is Einstein.

Proof. (1) From (2.3) we obtain $h_{j k}^{i}=b_{i k}^{j^{*}}$. Therefore we have

$$
\begin{aligned}
\operatorname{tr}\left(\sum A_{i^{*}}^{2}\right)^{2} & =\sum \operatorname{tr} A_{i^{*}}^{2} A_{j^{*}}^{2}=\sum b_{k l}^{i^{*}} b_{l m}^{i^{*}} b_{m b}^{j^{*}} b_{b k}^{j^{*}} \\
& =\sum b_{i l}^{k^{*}} b_{l i}^{m^{*}} b_{j b}^{m^{*}} b_{b j}^{k^{*}}=\sum\left(\operatorname{tr} A_{k^{*}} A_{m^{*}}\right)^{2} .
\end{aligned}
$$

(2) Since $M$ is Einstein so that $S=\rho g / n$ from (3.4) ${ }_{m}$ we have

$$
\sum A_{i^{*}}^{2}=(1 / 4(n-1) \tilde{c}-\rho / n) I=\|\sigma\|^{2} I / n, \text { or } \sum b_{j k}^{i^{*}} b_{k l}^{i^{*}}=\|\sigma\|^{2} \delta_{j l} / n \text {. }
$$

By the same argument as in (1), this is equivalent to

$$
\sum h_{i k}^{j^{*}} b_{k i}^{l^{*}}=\|\sigma\|^{2} \delta_{j l} / n \text { or } \operatorname{tr} A_{j^{*}} A_{l^{*}}=\|\sigma\|^{2} \delta_{j l} / n \text {. Q.E.D. }
$$

4. Some results on totally real minimal submanifolds in a complex space form.

Theorem 4.1. Let $M$ be an n-dimensional compact totally real minimal submanifold immersed in $\tilde{M}_{n}(\tilde{c}), \tilde{c}>0$. If

$$
\|\sigma\|^{2}<\frac{n(n+1)}{4(2 n-1)} \tilde{c} \text { or equivalently } \rho>\frac{n^{2}(n-2)}{2(2 n-1)} \tilde{c},
$$

then $M$ is totally geodesic.

Proof. An algebraic lemma of Chern, do Carmo and Kobayashi (Lemma 1 in [1]) implies $\operatorname{tr}\left(A_{i} A_{j^{*}}-A_{j} A_{i} A\right)^{2} \geq-2 \operatorname{tr} A_{i}^{2}{ }^{*} \operatorname{tr} A_{j}{ }^{2}$. Therefore, from Corollary 3.6, we have 


$$
\begin{aligned}
1 / 2 \Delta\|\sigma\|^{2} & \geq-2 \sum_{i \neq j} \operatorname{tr} A_{i^{*}}^{2} \operatorname{tr} A_{j^{*}}^{2}-\sum\left(\operatorname{tr} A_{i^{*}} A_{j^{*}}\right)^{2}+1 / 4(n+1) \tilde{c}\|\sigma\|^{2} \\
& =n^{-1} \sum_{i<j}\left(\operatorname{tr} A_{i^{*}}^{2}-\operatorname{tr} A_{j^{*}}^{2}\right)^{2}-\left(2-n^{-1}\right)\left(\sum \operatorname{tr} A_{i *}^{2}\right)^{2}+1 / 4(n+1) \tilde{c}\|\sigma\|^{2} \\
& \geq-\left(2-n^{-1}\right)\|\sigma\|^{4}+1 / 4(n+1) \tilde{c}\|\sigma\|^{2} \\
& =\frac{2 n-1}{n}\|\sigma\|^{2}\left(\frac{n(n+1)}{4(2 n-1)} \tilde{c}-\|\sigma\|^{2}\right) \geq 0 .
\end{aligned}
$$

Hence, by a well-known theorem of E. Hopf, $\Delta\|\sigma\|^{2}=0$ so that $\|\sigma\|=0$. This implies that $M$ is totally geodesic. Q.E.D.

As a special case of Theorem 4.1, we have [5]

Corollary 4.2. Let $M$ be a compact totally real minimal surface immersed in $\tilde{M}_{2}(\tilde{c}), \tilde{c}>0$. If the Gauss curvature of $M$ is positive, then $M$ is totally geodisic.

The following theorem is a generalization of Houh's result [3].

Theorem 4.3. Let $M$ be an n-dimensional totally real minimal submanifold immersed in $\tilde{M}_{n}(\tilde{c})$. If the sectional curvature of $M$ is constant, say $c$, then either $c=1 / 4 \tilde{c}^{n}$ (i.e., $M$ is totally geodesic) or $c \leq 0$.

Proof. Since $K=c$ so that $\rho=n(n-1) c$, from $(3.5)_{m}$ we have $c \leq 1 / 4 \tilde{c}$. Moreover, since $R_{j k l}^{i}=c\left(\delta_{i k} \delta_{j l}-\delta_{i l} \delta_{j k}\right)$, from (3.2), we have

$$
\sum\left(b_{i k}^{b^{*}} b_{j l}^{b^{*}}-b_{i l}^{b^{*}} b_{j k}^{b^{*}}\right)=(c-1 / \tilde{4})\left(\delta_{i k} \delta_{j l}-\delta_{i l} \delta_{j k}\right) .
$$

Multiplying $\Sigma b_{i l}^{m^{*}} b_{j k}^{m^{*}}$ on both sides of this equation and summing with respect to $i, j, k$ and $l$, we have

$$
\sum \operatorname{tr}\left(A_{b^{*}} A_{m^{*}}\right)^{2}-\sum\left(\operatorname{tr} A_{b^{*}} A_{m^{*}}\right)^{2}=(c-1 / 4 \tilde{c})\|\sigma\|^{2} .
$$

This, together with Corollary 3.6 and Lemma 3.7, implies $\left\|\nabla^{\prime} \sigma\right\|^{2}-n\left(n^{2}-1\right) c(c-1 / 4 \tilde{c})$, from which we deduce that either $c=1 / 4 \tilde{c}$ or $c \leq 0$. Q.E.D.

The following result is an immediate consequence of Theorem 4.3.

Theorem 4.4. Let $M$ be an n-dimensional totally real minimal submanifold immersed in $\tilde{M}_{n}(\tilde{c})$. If

(1) the sectional curvature $K$ of $M$ is constant,

(2) the second fundamental form of the immersion is parallel, then $M$ is either totally geodesic $(K=1 / 4 \tilde{c})$, or flat.

5. Totally real surfaces. Let $M$ be a surface (i.e., 2-dimensional Riemannian manifold) immersed in a $(1+p)$-dimensional complex space form $\widetilde{M}_{1+p}(\tilde{c})$. 
By considering the two-fold covering if necessary, we can assume that $M$ is orientable. It is well known that $M$ can be covered by a family of local isothermal coordinate systems. Let $\left(x_{1}, x_{2}\right)$ be a local isothermal coordinate system such that $g=E\left(d x_{1}^{2}+d x_{2}^{2}\right)$. Put $X_{i}=\partial / \partial x_{i}$ and $\sigma_{i j}=\sigma\left(X_{i}, X_{j}\right)$ for $i, j=1,2$. Then the equation of Codazzi gives

$$
\left\{\begin{array}{l}
\left(\tilde{R}\left(X_{1}, X_{2}\right) X_{1}\right)^{\perp}=D_{X_{2}}\left(\sigma_{11}-\sigma_{22}\right) / 2-D_{X_{1}} \sigma_{12}+E D_{X_{2}} H, \\
\left(\tilde{R}\left(X_{1}, X_{2}\right) X_{2}\right)^{\perp}=D_{X_{1}}\left(\sigma_{11}-\sigma_{22}\right) / 2+D_{X_{2}} \sigma_{12}-E D_{X_{1}} H .
\end{array}\right.
$$

This, combined with Proposition 3.1, implies the following.

Proposition 5.1. Let $M$ be a surface immersed in $\tilde{M}_{1+p}(\tilde{c})$ with $\tilde{c} \neq 0$. Then $M$ is a bolomorpbic or a totally real surface in $\tilde{M}_{1+p}(\tilde{c})$ if and only if the second fundamental form of the immersion satisfies the differential equations

$$
\left\{\begin{array}{l}
D_{X_{2}}\left(\sigma_{11}-\sigma_{22}\right) / 2-D_{X_{1}} \sigma_{12}+E D_{X_{2}}{ }^{H}=0 \\
D_{X_{1}}\left(\sigma_{11}-\sigma_{22}\right) / 2+D_{X_{2}} \sigma_{12}-E D_{X_{1}}{ }^{H}=0
\end{array}\right.
$$

$A$ unit normal vector field $\xi$ is called

(1) isoperimetric if $\operatorname{tr} A_{\xi}$ is constant,

(2) umbilical if $A_{\xi}$ is proportional to $I$,

(3) umbilic-free if $A_{\xi}$ is not proportional to $I$ everywhere,

(4) geodesic if $A_{\xi}=0$.

Proposition 5.2. Let $M$ be a bolomorpbic or totally real surface immersed in $\tilde{M}_{1+p}(\tilde{c})$. If $\xi$ is parallel and isoperimetric, then the function

$$
\phi_{\xi}=1 / 2 \tilde{g}\left(\sigma_{11}-\sigma_{22}, \xi\right)-\sqrt{-1} \tilde{g}\left(\sigma_{12}, \xi\right)
$$

is analytic in $z=x_{1}+\sqrt{-1} x_{2}$, where $\left(x_{1}, x_{2}\right)$ is a local isothermal coordinate system.

Proof. Taking the scalar product of the both sides of $(5.1)$ with $\xi$, we can see that $\phi_{\xi}$ satisfies the Cauchy-Riemann equation so that $\phi_{\xi}$ is analytic.

Proposition 5.3. Let $M$ be a topological sphere which is a totally real surface immersed in $\tilde{M}_{1+p}(\tilde{c})$. If a unit normal vector field $\xi$ is parallel and isoperimetric, then $\xi$ is umbilical.

Proof. Using the holomorphic function $\phi_{\xi}$ given in Proposition 5.2, we can define a (global) quadratic differential $\Phi$ on $M$ which is given locally by $\Phi=\phi_{\xi} d z^{2}$. By Riemann-Roch theorem, we have $\Phi \equiv 0$. This implies that $\xi$ is umbilical. 
Remark. Let $M^{\prime}$ be a totally umbilical (resp. totally geodesic) real hypersurface of $\tilde{M}_{1+p}(\tilde{c})$ and $M$ be a surface in $M^{\prime}$. Then a unit normal vector field of $M^{\prime}$ in $\tilde{M}_{1+p}(\tilde{c})$, restricted to $M$, is parallel and umbilical as a normal vector field of $M$ in $\tilde{M}_{1+p}(\tilde{c})$.

Theorem 5.4. Let $M$ be a compact totally real surface immersed in $\tilde{M}_{1+p}(\tilde{c})$. If

(i) the Gauss curvature does not change sign,

(ii) there exists a parallel umbilic-free isoperimetric unit normal vector field, then $M$ is flat.

Proof. Proposition 5.2 implies that the function $\phi_{\xi}$ is analytic. Therefore $\log \left|\phi_{\xi}\right|^{2}$ is harmonic, i.e., $\Delta \log \left|\phi_{\xi}\right|^{2}=0$, where $\Delta$ denotes the Laplacian formed with $g$. Since $\left|\phi_{\xi}\right|^{2}=E^{2}\left\{1 / 4\left(\operatorname{tr} A_{\xi}\right)^{2}-\operatorname{det} A_{\xi}\right\}$ and $\xi$ is umbilic-free, we have

$$
\Delta \log E^{2}=-\Delta \log \left\{1 / 4\left(\operatorname{tr} A_{\xi}\right)^{2}-\operatorname{det} A_{\xi}\right\} .
$$

Since the Gauss curvature $K$ of $M$ is given by $K=-\left(\Delta \log E^{2}\right) / 4 E$, we have

$$
K=(1 / 4 E) \Delta \log \left\{1 / 4\left(\operatorname{tr} A_{\xi}\right)^{2}-\operatorname{det} A_{\xi}\right\} \text {. }
$$

Therefore a theorem of $\mathrm{E}$. Hopf, together with the assumption that $K \geq 0$ or $K \leq 0$, implies $K \equiv 0$.

\section{BIBLIOGRAPHY}

1. B. Y. Chen, Geometry of submanifolds, Marcel-Dekker, New York, 1973.

2. S. S. Chern, M. P. doCarmo and S. Kobayashi, Minimal submanifolds of a sphere with second fundamental form of constant length, Functional Analysis and Related Fields (Proc. Conf. for M. Stone, Univ. Chicago, Chicago, Ill., 1968), Springer, New York, 1970, pp. 59-75. MR 42 \#8424.

3. C. S. Houh, Some totally real minimal surfaces in $C P^{2}$, Proc. Amer. Math. Soc. 40 (1973), 240-244.

4. K. Ogiue, On invariant immersions, Ann. Mat. Pura Appl. (4) 80 (1968), 387-397. MR $39 \# 6207$.

5. S. T. Yau, Submanifolds with constant mean curvature. I (to appear).

DEPARTMENT OF MATHEMATICS, MICHIGAN STATE UNIVERSITY, EAST LANSING, MICHIGAN 48104 